\title{
Salivary Gland Side Effects Commonly Develop Several Weeks After Initial Radioactive Iodine Ablation
}

Ravinder K. Grewal, Steven M. Larson, Christina E. Pentlow, Keith S. Pentlow, Mithat Gonen, Rebecca Qualey, Lauren Natbony, and R. Michael Tuttle

Memorial Sloan-Kettering Cancer Center, New York, New York

Salivary gland side effects (SSEs) can be a source of significant morbidity in thyroid cancer patients receiving radioactive iodine (RAI) for remnant ablation or therapy. However, the incidence, time course, and ultimate resolution of SSEs that develop in the first few months after a single administered activity of RAI for remnant ablation has not be adequately defined. Methods: We retrospectively reviewed the clinical records of patients after RAI remnant ablation (RRA) to determine the incidence of salivary gland-related side effects reported within the first year of RRA, the dose-response relationship between administered activity and specific SSEs, and the incidence of specific SSEs based on the method of preparation for remnant ablation (recombinant human thyroid-stimulating hormone [rhTSH] vs. traditional thyroid hormone withdrawal [THW]). Results: SSEs were reported within the first year of RAl ablation in 39\% of a cohort of 262 patients (66\% women, $93 \%$ papillary thyroid cancer; median dose, $5,217 \mathrm{MBq}[141 \mathrm{mCi}]$ ). Persistent side effects were noted after a median of $7 \mathrm{y}$ in $5 \%$ or less of the entire cohort. However, when side effects developed in patients during the first year, the incidence of persistence of the symptom at last follow-up ranged from $5 \%$ to $13 \%$. A statistically significant dose response was seen between administered activity of RAl and development of salivary gland swelling ( $P=0.001$, logistic dose-response curve) but not with dry mouth $(P=0.63)$, altered taste $(P=0.27)$, or salivary gland pain $(P=0.152)$. SSEs developed in $14 \%$ of patients receiving administered activities of $1,110 \mathrm{MBq}(30 \mathrm{mCi})$; administered activities of $2,775 \mathrm{MBq}(75 \mathrm{mCi})$ or more were associated with symptoms in $40 \%$ of patients $(P=0.046)$. Despite receiving a statistically higher administered activity $(5,661 \pm 2,997 \mathrm{MBq}$ $[153 \pm 81 \mathrm{mCi}]$ for THW vs. 4,958 $\pm 2,294 \mathrm{MBq}[134 \pm 62$ $\mathrm{mCi}$ ] for rhTSH), THW was associated with a lower rate of salivary gland swelling than the rhTSH preparation $(20 \%$ vs. $10 \%$; $P=$ 0.017 ), without differences in the development of dry mouth, altered taste, or salivary gland pain. Conclusion: Although SSEs occurred in $39 \%$ of patients after routine RRA, they were usually transient, so that the overall incidence of persistent side effects at a median follow-up of $7 \mathrm{y}$ was only $5 \%$. Even though the risk for persistent side effects is rather small, these data do emphasize the need to select patients carefully for RRA who are thought to be at moderate to high risk for recurrence and to use the min-

Received Dec. 22, 2008; revision accepted Jun. 23, 2009.

For correspondence or reprints contact: Ravinder K. Grewal, Division of Nuclear Medicine, Department of Radiology, P.O. Box 77, 1275 York

Ave., Memorial Sloan-Kettering Cancer Center, New York, NY 10065. E-mail: grewalr@mskcc.org

COPYRIGHT $\odot 2009$ by the Society of Nuclear Medicine, Inc. imally effective dose of RAl activity, in an attempt to maximize the potential benefit while minimizing the risk for adverse events for an individual patient.

Key Words: dose response; thyroid cancer; salivary gland; toxicities

J Nucl Med 2009; 50:1605-1610

DOI: 10.2967/jnumed.108.061382

$\mathbf{R}$ adioactive iodine (RAI) therapy is one of the important treatment modalities in the management of differentiated thyroid cancer $(1,2)$. RAI remnant ablation (RRA) has been demonstrated to decrease both risk of recurrence and disease-specific mortality in high-risk patients (3). Furthermore, RAI therapy is often an effective tool in the management of metastatic or locally invasive differentiated thyroid cancer $(4,5)$.

Although high cumulative administered activities have been associated with an increased risk of secondary malignancies (6), most authorities agree that life-threatening long-term complications from the usual activities of RAI administered for remnant ablation are rare. A wide spectrum of salivary gland complaints, ranging from mild transient discomfort to permanent xerostomia and tooth decay, has been described after RAI therapy. Fortunately, the common mild salivary gland side effects (SSEs) (such as minor discomfort and swelling of the parotid and submandibular salivary glands or minor discomfort in the region of the thyroid bed) that develop within a day or two after RAI administration are almost always transient and rarely develop into significant clinical problems. However, even relatively low administered activities of RAI used for remnant ablation have been associated with the more clinically significant side effects of xerostomia, salivary gland pain and swelling, dry eyes, excessive tearing, or alterations in taste in as many as $20 \%-30 \%$ of patients $(7-10)$. Fortunately, these side effects are usually transient but may persist in as many as $15 \%$ of patients $(11,12)$.

In the last several years, recombinant human thyroidstimulating hormone (rhTSH) was approved for use in RAI 
ablation of thyroid cancer patients without evidence of distant metastasis in both Europe and the United States. As would be expected, RAI is cleared from the blood and body more rapidly after rhTSH preparation than after traditional thyroid hormone withdrawal (THW) $(13,14)$. Because the side effects of RAI may be related both to administered activity and to cumulative dose, we hypothesized that the more rapid blood and whole-body clearance of RAI associated with rhTSH preparation for RRA would be associated with a decrease in radiation exposure to the salivary glands and potentially less salivary gland dysfunction than traditional THW. A recent study did suggest that the early, transient salivary gland symptoms developing within the first week after RAI ablation were less commonly seen after preparation with rhTSH $(9 / 30$ [30\%]) than after preparation with THW (35/60 [58\%]). However, the risk of persistent pain or xerostomia was similar in both groups (3\% with rhTSH and $4.6 \%$ with THW (15)).

To better define the incidence, time course, and ultimate resolution of SSEs that develop after a single administered activity of RAI for remnant ablation, we retrospectively analyzed the incidence of clinically significant salivary gland symptoms that developed within the first year after routine RRA in a consecutive series of thyroid cancer patients treated at our institution using either rhTSH-stimulated ablation or traditional THW.

\section{MATERIALS AND METHODS}

We retrospectively analyzed the nursing and physicians' medical records for patients undergoing radioiodine ablation for remnant thyroid tissue after thyroidectomy at our institution between 1995 and 2003. The usual follow-up for patients at our center is outpatient clinic visits every 6 mo for the first $2-3$ y after ablation. Therefore, data were generally obtained during these 6-mo follow-up visits or as reflected in phone call conversations that often occurred at more frequent intervals between the visits as symptoms developed.

The primary endpoints of this study were symptoms of dry mouth of any duration, alterations in taste that persisted for more than $2 \mathrm{mo}$, and any salivary gland pain or swelling that developed between 2 wk and 1 y after RRA. The presence, absence, resolution, date of onset, and date of resolution of each of these individual symptoms were documented. The time of follow-up for these patients was until the last clinical follow-up or until the subsequent RAI therapy dose. Patients were excluded if they had a history of salivary gland dysfunction, head and neck external beam radiation therapy, or corticosteroid use at the time of remnant ablation. This retrospective analysis was approved by our local institutional review board.

RRA was performed as previously described, using either traditional THW or rhTSH-stimulated preparation methods (16). In the hypothyroid protocol, levothyroxine was withdrawn for $6 \mathrm{wk}$ and triiodothyronine was withdrawn for $2 \mathrm{wk}$ before ablation. The rhTSH group received $0.9 \mathrm{mg}$ of rhTSH (Thyrogen; Genzyme Corp.) intramuscularly on days 1 and 2, with RAI administered on day 3. After the administration of radioiodine, patients were instructed to suck on sour candy starting an hour or so after dosing and continuing for $24-48 \mathrm{~h}$. They were also encouraged to drink lots of fluids but otherwise no specific therapy was given unless they became symptomatic, when nonsteroidal antiinflammatory medications were used. Oral glucocorticoids were used only for severe complaints unresponsive to nonsteroidal antiinflammatory medications.

\section{Statistical Analysis}

Data are presented as means, with SD and median values given when appropriate. Categoric comparisons were performed with the Fisher exact test, and means were compared between groups with an independent-samples $t$ test. The dose relationship between RAI administered activity and the development of symptoms was investigated using a logistic dose-response curve, which was estimated separately for each symptom using maximum likelihood. Analysis was performed using SPSS software (version 16.0.1; SPSS Inc.) or SAS software (version 9.2; SAS Institute).

\section{RESULTS}

\section{Study Cohort}

Complete data were available on 262 patients $(66 \%$ women; mean age, $45 \pm 16$ y) who underwent routine RRA (mean administered activity, 5,254 $\pm 2,627 \mathrm{MBq}$ [142 $\pm 71 \mathrm{mCi}]$; median, 5,217 MBq [141 mCi]) after total thyroidectomy using either THW (44\%) or rhTSH stimulation $(56 \%)$. Most of the patients had papillary thyroid cancer (93\%), with 58\% having stage I disease according to the staging system of the American Joint Committee on Cancer (Table 1).

\section{Development and Resolution of SSEs}

During the first year after RRA, 39\% (101/262) of patients reported at least 1 side effect that was likely to have been related to the RAI treatment (Table 2). The most commonly reported side effects included dry mouth $(17 \%)$, salivary gland swelling (16\%), altered taste for more than 2 mo (13\%), salivary gland pain $(6 \%)$, and excess tearing from tear-duct blockage (1\%).

Only 13 of the 262 patients (5\%) had complaints of SSEs at a median follow-up of $7 \mathrm{y}$ after RRA. When analysis was based on the entire treatment cohort, persistent dry mouth was noted at the time of last follow-up in only $2 \%(6 / 262)$, salivary gland swelling in $0.8 \%(2 / 262)$, altered taste in $1 \%$ (3/262), salivary gland pain in $0.4 \%(1 / 262)$, and tear-duct blockage in $0.8 \%(2 / 262)$ of the patients who underwent RRA.

However, for patients in whom side effects developed during the first year after RRA, symptoms did not resolve after a median follow-up of $7 \mathrm{y}$ in $13 \%$ (6/45) with dry mouth, $5 \%(2 / 41)$ with salivary gland swelling, 9\% (3/35) with alterations in taste, and 6\% (1/16) with salivary gland pain. Furthermore, the symptoms persisted for a median of 7-16 mo, depending on the specific complaint. Excess tearing from tear-duct blockage persisted in both patients until surgically corrected ( 2 and 4 y after RRA).

\section{Predictors of Side Effect Development}

When those patients in whom side effects developed (dry mouth, altered tasted, and salivary gland pain or swelling) 
TABLE 1. Demographics Table

\begin{tabular}{|c|c|}
\hline Variable & Data \\
\hline Age $(y)$ & $45 \pm 16$ \\
\hline Median & 44 \\
\hline Range & $13-89$ \\
\hline \multicolumn{2}{|l|}{ Sex } \\
\hline Female & $66 \%$ \\
\hline Male & $34 \%$ \\
\hline $\begin{array}{l}\text { RAl-administered } \\
\text { activity (MBq [mCi]) }\end{array}$ & $5,254 \pm 2,627(142 \pm 71)$ \\
\hline Median & $5,217(141)$ \\
\hline Range & $1,073-16,650(29-450)$ \\
\hline Thyroid bed uptake (\%) & $2 \pm 4$ \\
\hline Median & 0.7 \\
\hline Range & $0-29$ \\
\hline Follow-up years & $7 \pm 2$ \\
\hline Median & 7 \\
\hline Range & $5-12$ \\
\hline \multicolumn{2}{|l|}{ Histology } \\
\hline Papillary & $93 \%$ \\
\hline Follicular & $4 \%$ \\
\hline Hurthle & $3 \%$ \\
\hline \multicolumn{2}{|l|}{$\mathrm{T}$} \\
\hline 1 & $18 \%$ \\
\hline 2 & $30 \%$ \\
\hline 3 & $23 \%$ \\
\hline 4 & $20 \%$ \\
\hline Unknown & $8 \%$ \\
\hline \multicolumn{2}{|l|}{$\mathrm{N}$} \\
\hline Absent & $41 \%$ \\
\hline Present & $45 \%$ \\
\hline Unknown & $14 \%$ \\
\hline \multicolumn{2}{|l|}{ M } \\
\hline 0 & $86 \%$ \\
\hline 1 & $11 \%$ \\
\hline Unknown & $3 \%$ \\
\hline \multicolumn{2}{|l|}{ Stage } \\
\hline I & $58 \%$ \\
\hline II & $9 \%$ \\
\hline III & $17 \%$ \\
\hline IV & $8 \%$ \\
\hline Unknown & $8 \%$ \\
\hline Initial surgery: total thyroidectomy & $100 \%$ \\
\hline \multicolumn{2}{|l|}{ Preparation for ablation } \\
\hline Hypothyroid & $44 \%$ \\
\hline rhTSH & $56 \%$ \\
\hline
\end{tabular}

after RRA were compared with those patients in whom side effects did not develop, there were no significant differences in age at ablation $(46 \pm 17$ y vs. $45 \pm 15$ y), RAI administered activity $(5,476 \pm 2,627 \mathrm{MBq}$ vs. $5,106 \pm$ $2,664 \mathrm{MBq}[148 \pm 71 \mathrm{mCi}$ vs. $138 \pm 72 \mathrm{mCi}]$ ), thyroid bed uptake before RRA ( $2 \%$ vs. $2.5 \%$ ), duration of follow-up $(7.1 \pm 1.5$ y vs. $7.5 \pm 1.7$ y), stage of disease, or primary tumor histology. Women were more likely to report an SSE than men ( $43 \%$ vs. $29 \%, P=0.017)$.

When symptoms were analyzed individually, patients in whom salivary gland swelling developed received a significantly higher administered activity of RRA than those in whom this symptom did not develop $(6,475 \pm 2,849 \mathrm{MBq}$ vs. $5,069 \pm 2,553 \mathrm{MBq}[175 \pm 77 \mathrm{mCi}$ vs. $137 \pm 69 \mathrm{mCi}]$, $P=0.01)$. However, there was no significant difference in administered activities between those in whom dry mouth $(5,365 \pm 2,442 \mathrm{MBq}$ vs. $5,291 \pm 2,701 \mathrm{MBq}[145 \pm 66$ mCi vs. $143 \pm 73 \mathrm{mCi}])$, alterations in taste $(5,032 \pm 2,331$ MBq vs. $5,328 \pm 2,701 \mathrm{MBq}[136 \pm 63 \mathrm{mCi}$ vs. $144 \pm 73$ $\mathrm{mCi}])$, or salivary gland pain $(6,179 \pm 2,590 \mathrm{MBq}$ vs. $5,217 \pm 2,627 \mathrm{MBq}[167 \pm 70 \mathrm{mCi}$ vs. $141 \pm 71 \mathrm{mCi}]$ ) did or did not develop. The impact of administered activity on the subsequent development of side effects is more easily appreciated when the data are presented as categories that approximate the empiric dosing recommendations commonly used in practice (Fig.1). As expected, the lowest overall risks of side effects were associated with administered activities in the $1,110 \pm 370 \mathrm{MBq}(30 \pm 10 \mathrm{mCi})$ range. SSEs developed in $14 \%$ of patients receiving administered activities of $1,110 \mathrm{MBq}(30 \mathrm{mCi})$, and administered activities of $2,775 \mathrm{MBq}(75 \mathrm{mCi})$ or more were associated with symptoms in $40 \%$ of patients $(P=0.046)$. The risk of salivary gland swelling was in the $6 \%-8 \%$ range with administered activities ranging from 1,110 to $3,700 \mathrm{MBq}(30-100 \mathrm{mCi})$ but increased to $17 \%$ with activities ranging from 4,625 to $5,550 \mathrm{MBq}(125-150$ $\mathrm{mCi}$ ), with no further increase in the risk with single administered activities exceeding 6,105 MBq (165 mCi).

A statistically significant dose response was seen between administered activity of RAI and development of salivary gland swelling $(P=0.001$, logistic dose-response curve) but not with dry mouth $(P=0.63)$, altered taste $(P=0.27)$, or salivary gland pain $(P=0.152)$. Furthermore, there was no association between administered dose and resolution of any of the 4 major symptoms examined in this study (data not shown).

Our analysis of a potential effect of preparation method on the likelihood of side effects was complicated by the finding of a statistically higher administered activity in the hypothyroid withdrawal group than in the rhTSH-stimulated RRA ablation cohort $(5,661 \pm 2,997 \mathrm{MBq}$ vs. 4,958 \pm $2,294 \mathrm{MBq}[153 \pm 81 \mathrm{mCi}$ vs. $134 \pm 62 \mathrm{mCi}], P=0.03$ ). Even though the difference in doses is relatively small, this is the range for which the risk of salivary gland swelling begins to increase (Fig. 1). Comparing patients prepared with hypothyroid withdrawal with those prepared with rhTSH, we found no significant difference in the development of dry mouth (13\% vs. $20 \%)$, altered taste (10\% vs. $16 \%)$, or salivary gland pain (4\% vs. $8 \%)$. In univariate analysis, contrary to what we expected, patients prepared with rhTSH had a higher risk of salivary gland swelling (20\%) than patients prepared with hypothyroid withdrawal (10\%, $P=0.017$ by Fisher exact). Because of the relatively small number of events within each outcome group, multivariate analysis to identify independent risk factors could not be performed.

\section{DISCUSSION}

With our focus on the potential side effects of RAI, retrospective chart review of the patients being seen in a 
TABLE 2. Development and Resolution of Side Effects

\begin{tabular}{lcccc} 
& & & \multicolumn{2}{c}{ Time to resolution (mo) } \\
\cline { 2 - 4 } \multicolumn{1}{c}{ Side effect } & Developed side effect & Side effect resolved & Median & Mean \pm SD \\
Any symptom & $101 / 262(39 \%)$ & $249 / 262(95 \%)$ & 11 & $17 \pm 11$ \\
Dry mouth & $45 / 262(17 \%)$ & $39 / 45(87 \%)$ & 9 & $20 \pm 16$ \\
Altered taste $(>2$ mo) & $35 / 262(13 \%)$ & $32 / 35(91 \%)$ & 11 & $12 \pm 11$ \\
Salivary swelling & $41 / 262(16 \%)$ & $39 / 41(95 \%)$ & 7 & $9 \pm 6$ \\
Salivary pain & $16 / 262(6 \%)$ & $15 / 16(94 \%)$ & - \\
\hline Tearing & $2 / 262(1 \%)$ & $0 / 2(0 \%)$ & - \\
\hline
\end{tabular}

clinic showed $39 \%$ of patients (101/262) reporting the development of either dry mouth, alterations in taste that persisted more than 2 mo, or salivary gland pain or swelling within the first year of RRA. Fortunately, these symptoms resolved in most of these patients so that at the time of last follow-up less than $1 \%$ had persistent pain or swelling in the salivary glands and $2 \%$ had persistent dry mouth at the time of the last follow-up a median of $7 \mathrm{y}$ after ablation. However, in the patients in whom side effects developed in the first year, 13\% had persistent dry mouth, 9\% had persistent altered taste, $5 \%$ had persistent salivary gland swelling, and $6 \%$ had persistent salivary gland pain. Neither patient with blockage of the tear ducts had spontaneous resolution of the symptom. Our approach involves preselection of patients with moderate- to high-risk likelihood for recurrence based on epidemiologic experience of clinical and surgical findings at presentation (17).

Several previous studies have reported rates of SSEs ranging from $11.5 \%$ to $86 \%$ in patients receiving multiple doses of RAI. In general, in these studies higher cumulative activities were associated with increased risk of SSEs $(9,18-21)$. Two other prospective studies examined only acute side effects that developed in the first week after RAI therapy without long-term follow-up and are therefore not directly comparable to our data $(10,15)$.

Only 4 studies (Table 3) $(11,12,22,23)$ that would be comparable to our series have examined long-term SSEs with single-dose RAI. These studies have reported salivary gland toxicity rates in the range of $6.7 \%-33 \%$ in patients prepared with traditional THW. None of these series included patients prepared with rhTSH stimulation. Similar to our study, a dose-response relationship was seen in 2 of the studies $(11,23)$, demonstrating an increased risk of SSEs at administered activities greater than 11,100 MBq (300 mCi). The slightly higher incidence of side effects in our series is likely related to improved documentation associated with the standardized data collection forms completed by a trained thyroid cancer nurse or clinicians with an interest in potential RRA side effects.

Similar to previous studies, our data did demonstrate a dose-response relationship between administered activity of RAI and salivary gland swelling $(P=0.001$ by logistic doseresponse curve analysis). Administered activities of greater than $2,775 \mathrm{MBq}(75 \mathrm{mCi})$ were associated with more SSEs
FIGURE 1. Comparison of side effects with administered activity of ${ }^{131} \mathrm{I}$. Because administered activities tended to cluster around standardized empiric dosing amounts, we analyzed data based on usual administered activity levels \pm approximately $370 \mathrm{MBq}(10$ $\mathrm{mCi})$.

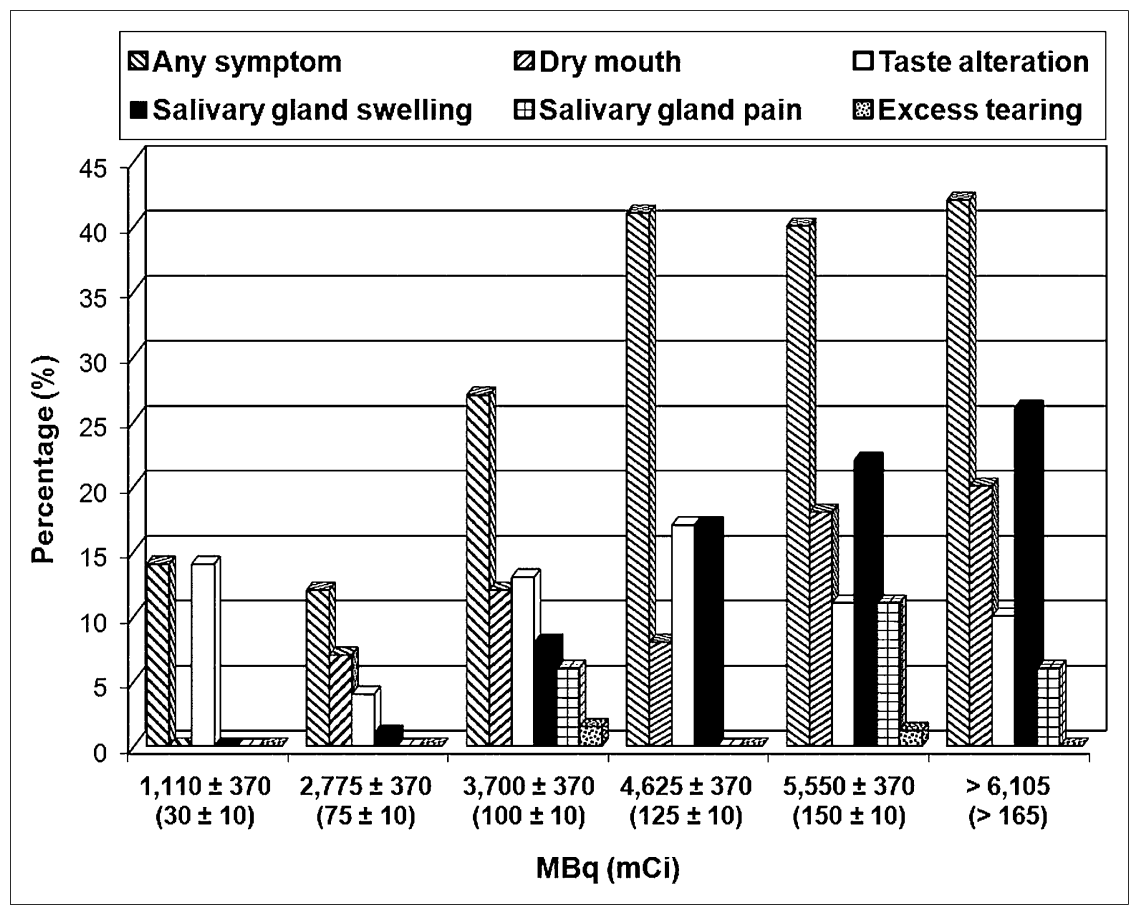


TABLE 3. Published Studies Examining Long-Term SSEs with Single-Dose RAI

\begin{tabular}{|c|c|c|c|c|c|c|c|c|}
\hline Study & $\begin{array}{l}\text { No. of } \\
\text { patients }\end{array}$ & $\begin{array}{l}\text { Method of } \\
\text { preparation }\end{array}$ & $\begin{array}{l}\text { Study } \\
\text { design }\end{array}$ & $\begin{array}{c}\text { First ablation } \\
\text { with }{ }^{131} \mathrm{I} \text {, median } \\
\text { dose range }(\mathrm{MBq})\end{array}$ & $\begin{array}{l}\text { Salivary } \\
\text { gland } \\
\text { toxicity (\%) }\end{array}$ & $\begin{array}{l}\text { Dose-response } \\
\text { relationship }\end{array}$ & $\begin{array}{l}\text { Follow-up } \\
\text { duration } \\
\text { (mo) }\end{array}$ & $\begin{array}{l}\text { Chronic } \\
\text { symptoms }\end{array}$ \\
\hline $\begin{array}{l}\text { Solans } \\
\text { et al. (11) }\end{array}$ & 79 & Hypothyroid & Prospective & $925-18,500(25-500)$ & 33 & Yes & 36 & $15 \%$ at $36 \mathrm{mo}$ \\
\hline Hyer et al. (12) & 76 & Hypothyroid & Prospective & $2,960-8,695$ (80-235) & 26 & Not reported & 12 & $20 \%$ at $12 \mathrm{mo}$ \\
\hline $\begin{array}{l}\text { Edmonds and } \\
\text { Smith (22) }\end{array}$ & 258 & Hypothyroid & Retrospective & $2,960-8,510(80-230)$ & 10 & $?$ & Unknown & - \\
\hline $\begin{array}{l}\text { Hoelzer } \\
\text { et al. (23) }\end{array}$ & 2,376 & Hypothyroid & Prospective & $999-11,988$ (27-324) & $6.79^{*}$ & Yes & Unknown & - \\
\hline
\end{tabular}

than administered activities of approximately $1,110 \mathrm{MBq}(30$ $\mathrm{mCi}$ ) (Fig. 1). In particular, the incidence of dry mouth appeared to increase at administered activities above 2,775 $\mathrm{MBq}(75 \mathrm{mCi})$, whereas the risk of salivary gland swelling appeared to increase at doses greater than $4,625 \mathrm{MBq}(125$ $\mathrm{mCi}$ ). Conversely, the symptoms of dry mouth, alterations of taste, and excess tearing appear to be much less dose-related, with similar risks across all dose ranges examined.

Zanzonico estimated the typical salivary gland dose (mean whole-gland dose) as $300 \mathrm{rad}$ for a $200-\mathrm{mCi}^{131}$ I treatment of metastatic thyroid cancer (24). Zanzonico presented a firstorder calculation (based on collection and counting of saliva samples from an ${ }^{131}$ I-treated thyroid cancer patient and a small-scale dosimetry analysis), indicating that the dose to the epithelial lining of the salivary gland ducts was probably at least 3 - to 4 -fold higher ( $>1,000 \mathrm{rad})$ than the mean gland dose. This study was based on the speculation that a 1,000rad-plus dose to the salivary duct lining is more consistent with sialadenitis than the mean salivary dose of only $300 \mathrm{rad}$. There is an apparent lack of relationship to the dosimetry of the salivary gland itself, and the probable target is the lining of the salivary gland ducts (P. Zanzonico, oral communication, December 1987).

A recent study by Rosario et al. (15) did suggest that the early, transient salivary gland symptoms developing within the first week after RAI ablation were less commonly seen after preparation with rhTSH $(9 / 30$ [30\%]) than after preparation with THW (35/60 [58\%]). However, the risk of persistent pain or xerostomia was similar in both groups (3\% with rhTSH and $4.6 \%$ with THW (15)). Therefore, it is possible that rhTSH-assisted remnant ablation may be associated with less early, transient toxicity than THW. However, the risk of long-term, persistent effect appears to be similar between rhTSH and THW in both our study and the Rosario experience.

In our study, we found that preparation with rhTSH had a higher risk of later-onset salivary gland swelling than preparation with THW. Hanscheid et al. (13) observed a longer effective half-life in normal thyroid remnants after rhTSH than after THW. However, it seems unlikely that the iodine kinetics of residual thyroid cells remaining after a total thyroidectomy would predict the kinetics of iodine retention within salivary gland tissues.

Contrary to our hypothesis, the more rapid clearance of RAI from blood and body seen with rhTSH preparation was not associated with a decrease in the risk of salivary gland pain or swelling, dry mouth, or alterations in taste. It is likely that the lack of difference between the 2 preparation methods was secondary to the rather large RAI administered activity used in this study $(5,291 \pm 2,627 \mathrm{MBq}$ $[143 \pm 71 \mathrm{mCi}])$. These larger RAI ablation doses were used in our early studies, with our more current studies suggesting that a median activity of approximately 3,700 $\mathrm{MBq}(100 \mathrm{mCi})$ is adequate for ablation of patients at moderate to high risk of recurrence without known metastatic disease (25). Even with a $25 \%$ more rapid clearance after rhTSH preparation, the high administered activities of RAI used in this study would still result in the salivary glands being exposed to an equivalent dose of RAI that was above the 2,775-MBq (75-mCi) administered activity level for development of dry mouth and near the $4,625-\mathrm{MBq}$ (125-mCi) level for developing salivary gland swelling. However, it is likely that the risk of SSEs may be lower when administered activities of 2,775-3,700 MBq (75-100 $\mathrm{mCi}$ ) are given after rhTSH preparation, because the equivalent dose would likely fall below the apparent threshold for significant salivary gland dysfunction.

Although the side effects tended to be associated with increasing administered activity, it is clear that in many patients given relatively large RAI doses, SSEs, if any, developed only minimally. Furthermore, even with rather small administered activities, significant SSEs developed in some patients. Therefore, important variables other than administered activity must be playing a major role in the likelihood of SSE development. These variables could include a difference in radiation sensitivity, actual dose to the salivary glands, hydration status, and use of sour candies or nonsteroidal inflammatory medications not reported to the treating physician. Future studies will be required to answer these questions. 


\section{CONCLUSION}

Although RAI is an important tool in the management of thyroid cancer, it is not without side effects. In this cohort, $39 \%$ of patients reported at least 1 SSE within the first year of RAI ablation. Although persistent complaints were reported in 5\% or less of the entire cohort of patients a median of $7 \mathrm{y}$ after ablation, the risk of having persistent symptoms ranged from 5\% to $13 \%$ in those patients in whom symptoms developed within the first year of ablation. An apparent dose-response relationship was seen in some, but not all, of the SSEs. With the relatively large administered activities of RAI used for ablation in this series, the more rapid wholebody clearance of RAI associated with rhTSH preparation was not associated with a decrease in SSEs. Future studies are needed to determine whether the SSE profile would be different between rhTSH preparation and THW at administered activities in the 1,850 - to $3,700-\mathrm{MBq}$ (50- to $100-\mathrm{mCi}$ ) range that is now more commonly used for RRA.

\section{REFERENCES}

1. Cooper DS, Doherty GM, Haugen BR, et al. Management guidelines for patients with thyroid nodules and differentiated thyroid cancer. Thyroid. 2006;16:109-142.

2. Tuttle RM, Leboeuf R, Martorella AJ. Papillary thyroid cancer: monitoring and therapy. Endocrinol Metab Clin North Am. 2007;36:753-778.

3. Mazzaferri EL, Kloos RT. Clinical review 128: current approaches to primary therapy for papillary and follicular thyroid cancer. $J$ Clin Endocrinol Metab. 2001;86:1447-1463.

4. Schlumberger MJ. Papillary and follicular thyroid carcinoma. $N$ Engl J Med. 1998;338:297-306.

5. Durante C, Haddy N, Baudin E, et al. Long-term outcome of 444 patients with distant metastases from papillary and follicular thyroid carcinoma: benefits and limits of radioiodine therapy. J Clin Endocrinol Metab. 2006;91:2892-2899.

6. Brown AP, Chen J, Hitchcock YJ, Szabo A, Shrieve DC, Tward JD. The risk of second primary malignancies up to three decades after the treatment of differentiated thyroid cancer. J Clin Endocrinol Metab. 2008;93:504-515.

7. Shen DH, Kloos RT, Mazzaferri EL, Jhian SM. Sodium iodide symporter in health and disease. Thyroid. 2001;11:415-425.

8. Mandel SJ, Mandel L. Radioactive iodine and the salivary glands. Thyroid. 2003;13:265-271.
9. Allweiss P, Braunstein GD, Katz A, Waxman A. Sialadenitis following I-131 therapy for thyroid carcinoma: concise communication. J Nucl Med. 1984;25: $755-758$.

10. Lin WY, Shen YY, Wang SJ. Short-term hazards of low-dose radioiodine ablation therapy in postsurgical thyroid cancer patients. Clin Nucl Med. 1996; 21:780-782.

11. Solans R, Bosch JA, Galofre P, et al. Salivary and lacrimal gland dysfunction (sicca syndrome) after radioiodine therapy. J Nucl Med. 2001;42:738-743.

12. Hyer S, Kong A, Pratt B, Harmer C. Salivary gland toxicity after radioiodine therapy for thyroid cancer. Clin Oncol (R Coll Radiol). 2007;19:83-86.

13. Hanscheid H, Lassmann M, Luster M, et al. Iodine biokinetics and dosimetry in radioiodine therapy of thyroid cancer: procedures and results of a prospective international controlled study of ablation after rhTSH or hormone withdrawal. J Nucl Med. 2006;47:648-654.

14. Menzel C, Kranert WT, Dobert N, et al. rhTSH stimulation before radioiodine therapy in thyroid cancer reduces the effective half-life of ${ }^{131} \mathrm{I}$. J Nucl Med. 2003;44:1065-1068.

15. Rosario PW, Borges MA, Purisch S. Preparation with recombinant human thyroid-stimulating hormone for thyroid remnant ablation with ${ }^{131} \mathrm{I}$ is associated with lowered radiotoxicity. J Nucl Med. 2008;49:1776-1782.

16. Robbins RJ, Larson SM, Sinha N, et al. A retrospective review of the effectiveness of recombinant human TSH as a preparation for radioiodine thyroid remnant ablation. J Nucl Med. 2002;43:1482-1488.

17. Tuttle RM, Leboeuf R, Shaha AR. Medical management of thyroid cancer: a risk adapted approach. J Surg Oncol. 2008;97:712-716.

18. Alexander C, Bader JB, Schaefer A, Finke C, Kirsch CM. Intermediate and longterm side effects of high-dose radioiodine therapy for thyroid carcinoma. $\mathrm{J} \mathrm{Nucl}$ Med. 1998;39:1551-1554.

19. Caglar M, Tuncel M, Alpar R. Scintigraphic evaluation of salivary gland dysfunction in patients with thyroid cancer after radioiodine treatment. Clin Nucl Med. 2002;27:767-771.

20. Van Nostrand D, Neutze J, Atkins F. Side effects of "rational dose" iodine-131 therapy for metastatic well-differentiated thyroid carcinoma. J Nucl Med. 1986; 27:1519-1527.

21. Malpani BL, Samuel AM, Ray S. Quantification of salivary gland function in thyroid cancer patients treated with radioiodine. Int J Radiat Oncol Biol Phys. 1996;35:535-540.

22. Edmonds CJ, Smith T. The long-term hazards of the treatment of thyroid cancer with radioiodine. Br J Radiol. 1986;59:45-51.

23. Hoelzer S, Steiner D, Bauer R, et al. Current practice of radioiodine treatment in the management of differentiated thyroid cancer in Germany. Eur J Nucl Med. 2000;27:1465-1472.

24. Zanzonico P. Radiation dose to patients and relatives incident to I131 therapy. Thyroid. 1997;7:199-204.

25. Tuttle RM, Brokhin M, Omry G, et al. Recombinant human TSH-assisted radioactive iodine remnant ablation achieves short-term clinical recurrence rates similar to those of traditional thyroid hormone withdrawal. J Nucl Med. 2008; 49:764-770. 